\title{
Competent Fuzzy Decision Making Algorithm using Hyper ETL in Data Mart
}

\author{
A. Prema \\ Assistant Professor, Department Of Computer Science, \\ Raja Doraisingam Government Arts College,Sivaganga, Tamilnadu,India, \\ Email Id : latharaman2012jr@gmail.Com
}

\begin{abstract}
In a period of scientific advancement, technical innovation and quicker development in modern computing, an unimaginable quantum of data is exchanged, processed, stored, referred to, analyzed, for various purposes. Be it any discipline, industry or branch of study managing data has become unwieldy. A proper organization of data will pave the way for easy access for various desired functions which will in turn determine the success of a company. In these days of an avalanche of data it has become imperative to initiate innovative methods. Great interest and utmost care is taken in this paper to suggest fuzzy optimization to achieve greater accurate and effective solutions. The growth in the number of business looking for data mart solutions, with the aim of adding major business gains, has generated the need for a decision-aid has come near in preferring the right data mart system. Due to the indefinite concept often denoted in decision-making procedure, to facilitate decision matrix analysis, with fuzzy optimization technique which scrutinize the best decision making for sales promotion to the various places. Fuzzy Optimization technique is the symmetric approach of decision making process which is used to demonstrate the maximum sales quantity depending on the places. This paper propose a new FDM (Fuzzy Decision Making) algorithm, to evaluate the performance of sales promotion using Hyper ETL (Extract, Transform and Load) in sales Data Mart.
\end{abstract}

Key Words- Fuzzy optimization, fuzzy decision making algorithm, data mart, Hyper ETL, Decision Matrix, Decision making.

\section{INTRODUCTION}

Traditional optimization techniques and methods have been successfully applied for many years. It is used to solve problems with a well-defined structure or configuration. Fuzzy optimization has received much attention with various models and methods, because it has been proposed by many researchers. Recent surveys on fuzzy optimization technique are being focused on special category of the fuzzy mathematical programming. They are extended to represent a type of approaches for symmetric mathematical programming models in the sense, the goals involves in the problem dealt with in a symmetric way with regard to fuzziness. Symmetric approaches are based on fuzzy decision. The fuzzy decision is defined as a fuzzy set of alternatives resulting from Union and Intersection. A Maximizing decision $\mathrm{X}^{*}$ is defined to be an alternative with the highest membership in the fuzzy decision D, i.e.,

$$
\mu \mathrm{D}\left(\mathrm{X}^{*}\right)=\underset{x \in X}{U} \mu D(x)
$$

The maximization decision $\mathrm{X}^{*}$ is the optimal solution for a sense that it can be interpreted with different ways, depending on the definitions of the operators $\cap$ and $\cup$. The operator $\cap$ may be extended to various forms of conjunctive operators, such as minimum operator, weighted sum of the goals, constraints, multiplication operator, mean value operator, bounded product and Hamcher's min operator. The selection of the operators depends on the preference of the decision-maker and the problem context. This approach provides a framework for solving fuzzy optimization techniques with fuzzy goals and fuzzy constraints and it is well known as the fundamental decision making under the fuzzy environment.

This approach can be applied to the cases with a single objective or multiple objectives in the forms of linearity or nonlinearity. This types of optimal solution to this approach can be expressed in the different forms such as fuzzy decision ie., maximizing decision and fuzzy optimal solution are depending on the operators and the interpretation applied. 
Section 2 of this paper deals with related work done in Data Mart, Hyper Extract, Transformation and Loading and Fuzzy, Section 3, explains the proposed work, in section 4, Experimental analysis, results are given respectively, and finally, section 5 presents a conclusion of this paper.

\section{RELATED WORKS}

Li Jain conquered the pathetic points of conventional Extract, Transform and Load tool's architecture and proposed a three layers architecture based on metadata. That builds ETL process more flexible, multipurpose and competent and then they designed and implemented a new ETL tool for drilling data ware house. A systematic review method is proposed to identify, extract and analyze the main proposals on developing conceptual ETL processes for Data Warehouse. The main proposals are identified and compared based on the features, activities and notation of ETL processes and concluded the study by reflecting on the approached being studied and providing an update skeleton for future study [7]

Sabir Asadullaev talked about centralized Extract, Transform and Load with related Data mart, Data warehouse and applications of data mart, data warehouse with integration bus and recommended data warehouse architecture [8]. Last few the years, data warehouse technology has been used for analysis and decision making in enterprises [4]. Different kinds of approaches for the integration of ETL tool in data warehouse have been proposed.

Shaker H. Ali El-Sappagh tried to navigate though the effort done to conceptualize abbreviations for ETL, DW, DM, OLP, on-line analytical processing, DS, ODS, and DSA [9]. A data warehouse gives a set of numeric values that are depends on group of input values in the form of dimensions [6]. Data Mart can hold information which addresses both strategic and tactical information and provides an information which allows key operating function effectively to manage performance. It unifies information from various databases into a single database. A data mart contains data from a particular business area and multiple data mart can form a data warehouse. Data marts are the cornerstones of the enterprise, and each unique knowledge data mart is maintained by a divisional or departmental group. The motives for building a data mart are specified below [14].

a) Improves end-user response time

b) Creates collective view by a group of users

c) Provides ease of creation

d) Easy access to frequently need data

e) Lower cost than implementing a full Data warehouse

Daniel Fasel reveals the uses of a fuzzy data warehouse approach to sustain the fuzzy analysis of a customer performance measurement. The potential of the fuzzy data warehouse approach is illustrated by using a concrete example of customer performance measurement for hearing instrument manufacture. A few for combining fuzzy concepts with the hierarchies of data ware house have been proposed. A method of summary can be guaranteed by using this approach in data ware house concepts improves information quality for the company. It provides broader possibilities to create indicators for customer performance measurement as in the example given. The proposed approach does not include fuzzy linguistic concepts directly in to the hierarchical structure of dimension or into fact tables of the data warehouse model and also explains how the fuzzy concepts can be aggregated over dimensions without having redefined the fuzzy sets in every degree of granularity [17]. Visualization should provide easy understanding of the result for fuzzy queries in the fuzzy data warehouse.

Owner Kaser et al., describes to apply the business intelligent techniques for the data warehousing and OLAP to the domain of text processing. A literary data warehouse is the conventional corpus but its data stored and organized in multidimensional stages, so as to promote efficient end user queries. This work increases the query engine, ETL process and the user interfaces. The extract, transform, load stage retains the information that are built by the data warehouse. The overall thought of applying OLAP to mythical data is promising. The initial custom engine is slow for production use, but until more optimization is endeavored, its assure is unclear [21]

A concrete ETL service framework was proposed and talked about metadata management service, metadata definition services, ETL transformation rules service, process definition service etc [3]. Two heuristic algorithms with insatiable characteristics were proposed to condense the execution cost of an ETL workflow [10]. Lunan Li has recommended to rigorously manage ETL by metadata repository and composes metadata easier to understand; therefore metadata management becomes more direct, simple and centered. Numeric values of a classical data warehouse can be complicated to realize for business users, or may be interpreted incorrectly. Therefore, for a more 
accurate interpretation of numeric values, business users require an analysis in meaningful non-numeric terms. However, if the transition between terms is crisp, true values cannot be measured and smooth transition between classes cannot take place [1]. Eventually, definition method and related algorithms of ETL rules are multiple data marts can form a data warehouse [5]. ETL is an authoritative meta data based procedure that extracts the data from source system and loads into the data warehouse and this process improves overall data quality and report ability.

Radhakrishnan and Sreekanth proposed a web based framework form for denoting the extraction of data from one or more data source and use transformation business logic and load the data within the data warehouse. This is fine starting point for collecting information in the exiting documentation for the system and also researching for ETL phase in web based scenario modeling in distributed environment which provides an effective evaluation results for diverse organization [22]. The models of the entire ETL process using UML because these structural and dynamic properties of an information system at the conceptual level are more likely than the immature approaches. It is more adaptable and is used to sustain trading corporation, banks, financials and human resource management system of an organization at diverse levels. The future track of this paper includes analyzing multimedia information source automating mechanisms for ETL process.

James F. Brule's demonstrates the fuzzy system is an substitute to traditional perceptions of set membership and logic the has its origin in ancient Greek philosophy and its applications are the leading edge of artificial intelligence and it nearby the foundation of fuzzy systems with formal mathematics [18]. It is used in many applications such as information retrieval system, a navigation system for automatic cars, a extrapolative fuzzy logic controller for automatic operation of trains, and laboratory water level controllers for ROBOT are welders, feature definition controllers for ROBOT vision, graphics controller for computerized police sketchers and more. Fuzzy systems together with fuzzy logic and fuzzy set theory provide a rich and meaningful addition to standard logic. The mathematics produced by theories is constant; a fuzzy logic may be a generalization of classic logic. Many systems may be modeled and event replicated with the help of fuzzy systems.

Lior Sapir et al., suggests that a data ware house is a special database used for storing business oriented information for future analysis and decision making. In business scenario, where a few of the data or the business attributes are fuzzy, it may be useful to construct a warehouse that can support the analysis of fuzzy data and also sketched the Kimball's methodology for the design of a data ware house can be extended to the construction of a fuzzy data warehouse. A case study which demonstrates a visibility of the most frequently used methodology today is Kimball's. It depicts the process of translating business data and prose into a dimensional model. It has several advantages, but one of them is that users can compose additional intuitive and painless to understand queries in a natural language. Defining fuzzy dimensions allows the user to describe the facts with abstract human concepts which are actually more realistic [20]. The fuzzy dimensions also permit more flexible and interesting filtering of the facts. We have demonstrated that fuzzy measures used with fuzzy aggregation operators allow the user to better understanding business and data ware house measures.

D. Ashok Kumar and M. C. Loraine elucidated modern electronic health records which are designed to capture and render vast quantities of clinical data during the health care prone. Utilization of data analysis and data mining method in medicine and health care is sparse. Medical data is one of the heavily and categorical types of data. A Dichotomous variable types of categorical data are binary categorical with zero and one. Binary data are the simplest form of data used for medical database in which close ended questions can be used. It is very efficient based on computational efficiency and has memory capacity to represent categorical type data. Data mining technique called clustering is involved here for dichotomous medical data due to its high dimensional and data scarceness. Usually the binary data clustering is done by using 0 and 1 as numerical value. The clustering is performed after transforming the binary data into real by wiener transformation. The suggested algorithm can be compatible for large medical and health binary data bases for determining the correction are the health disorders and symptoms observed [16].

Christ Sophie et al., presents that in the field of human resources: there is a growing trend towards moving from activity based functions to a more strategic, business oriented role. The data mart termed on the HR information needs is the preeminent solution to meet the objectives [15]. The main purpose of this paper is to explain how the SAS system can be applied in top of SAP R/3 HR, and attain real business profit with a very short time. It is also based on the practical experience at the Belgian Gas and electricity provider. The structure of this paper first explains the business functions that covers shortcomings of the system. The solution to short coming is explained and business objectives for the data mart are discussed. Finally, this paper explains the projected approach and focuses on the specific attention points when building a data mart. It provides end to end solution and data management facilities are probable to distribute quick end result to the end users.

Jeremy, Andeas et al., have built the dominant data marts that require minimal administration and are simple to change. This may seem like an impossible goal to anyone who has been involved in the usual complexity but there are a number of simple, practical concepts and methodologies that have been employed and tested over 
several years and the triumphant data warehouse implementation that are repeatable and are easy to understand [19]. For the purpose of data ware housing ETL is used to pull data from business systems into a database that are designed for analysis and reporting. Building data mart and ETL processes involves large volumes of complex business data and the easiest outcome is also complexity. But Lack of results are expected due to the easiest outcome of the more resources. It is also used to achieve powerful results with a short span of time is useful to users and fulfills the core requirement of effective visibility into their complex business data. Fuzzy union and intersection are used to take optimal solution [25].

Extract, Transform and Load (ETL) is a process that involves extracting data from product source, transforming it through encoded business rules to fit business needs, and loading it into the data warehouse from where reports are produced. One can modify the ETL jobs to outfit your specific business requirements. The three data base functions are combined into one tool that automates the process to drag data out of one database into another database [2]. The testing of ETL mainly deals with how, from, when, what and where we carry in our database. All tables except the reference table are moved to the Data warehouse using an ETL process [8]. Many tables are split into smaller tables in order to expedite queries. The ETL process [15] includes designing a objective, extracting data from sources, transforming data for the target, scheduling and monitoring processes, and managing the Business Intelligence environment.

The ETL tools [17] were created to advance and facilitate data warehouse. ETL eliminates the step of loading the text files into intermediate storage, saving significant space and time. Hyper ETL tool is used for managing metadata structure and improve an effectiveness of an ETL process. Hyper ETL reduces the transformation time than an existing one. This enrichment become wider the aggregation method, convey information intelligently and is useful for an effective decision making. The Hyper ETL process consists of the following steps[27]:

1. Extract the data from operational data source. Data extraction is one of the three main functionalities of the ETL tools. A main consideration to assess is the product's ability to extract from a variety of various data sources.

2. Create tables with related attributes based on user constraints.

3. Renovate it to robust operational needs. Generate the XML document file.

4. Create the Meta-Data for XML document File.

This Research work applys three protocols namely Oracle Database, XML Data File and JDBC. The Protocol will be part of the url attribute of the target or source node. Every transformation will have a source and target.

<source url="xml://localhost/etl/test.xml"“>

...

<target url="jdbc:oracle:thin:@localhost:1521:XE"

5. Remove the inconsistent data.

6. Split the table.

7. Allot the data.

8. Load it into the end target (ie) Pump the data into Oracle data warehouse. The loading phase is the last step of the ETL process. The information from data sources are loaded and stored in a form of tables. There are two types of tables in the database structure: Fact tables and Dimensions tables. Once the fact and dimension tables are loaded, it is time to improve the performance of the Business Intelligence data by creating Aggregates.

\section{Audit reports}

10. Publish is the manual step to recap the position of the previous day's.

\section{Archive}

12. Clean up (giving out data to advance its excellence) 


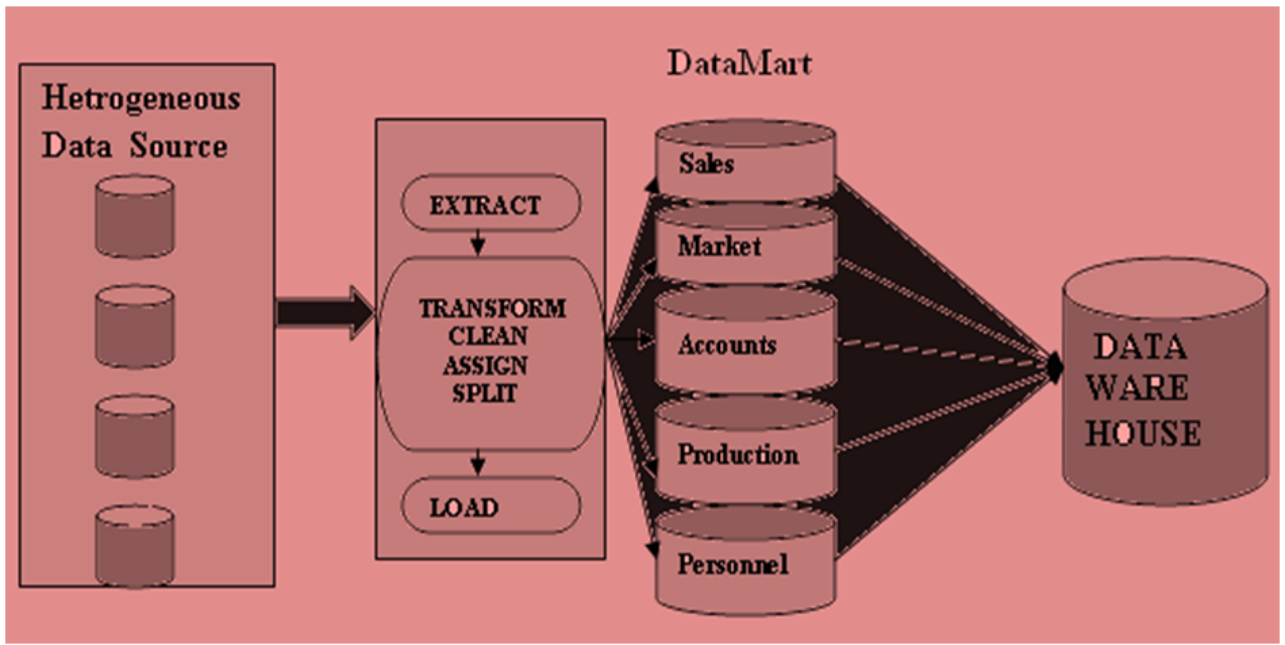

Figure 1: ETL Diagram [23]

Benefits of an ETL [16] Tool are given below:
a) To simplify the process of migrating data
b) To Store all data transformation logic/rules as Meta data
c) To Shrink cost and effort associated with building interfaces
d) To Standardize the method of data migration
e) To Enable Users, Managers and architects to understand, review, and modify the various interfaces.

\section{PROPOSED WORK}

The decision matrix is appeared to show the quantity of sales on different places based on the matrix, put the rank, depending on the rank, select the best movement of items and these items are used to produce the maximum movement of sales on particular places. Finally apply our proposed FDM algorithm which optimizes the maximum sales quantity in various places, 2450 real time Sales data are taken into the account for evaluating this hyper ETL [26]. The dataset is given below for reference.

Table 1: Real time sales Dataset

\begin{tabular}{|l|c|c|c|c|c|c|c|c|}
\hline $\begin{array}{l}\text { Place/ } \\
\text { Item }\end{array}$ & Madurai & Sivagangai & Virdhunagar & Theni & Manamadurai & Ramnad & Paramakudi & Sivakasi \\
\hline M12 & 200 & 100 & 100 & 42 & 65 & 70 & 35 & 50 \\
\hline N14 & 300 & 200 & 100 & 100 & 47 & 58 & 23 & 30 \\
\hline Q11 & 200 & 100 & 100 & 60 & 25 & 57 & 42 & 35 \\
\hline A12 & 250 & 250 & 150 & 150 & 100 & 70 & 82 & 95 \\
\hline C14 & 40 & 80 & 40 & 40 & 20 & 30 & 45 & 30 \\
\hline D14 & 144 & 192 & 144 & 96 & 40 & 48 & 30 & 40 \\
\hline F15 & 160 & 40 & 30 & 25 & 20 & 35 & 30 & 32 \\
\hline H11 & 50 & 150 & 50 & 40 & 35 & 25 & 37 & 30 \\
\hline
\end{tabular}

Using the decision matrix method, we rank the places and items by applying Laplace method According to the decision matrix analysis, we chose best two items(A12 and N14) and two places(Madurai and Sivagangai). 
Table 2: Decision Analysis Matrix

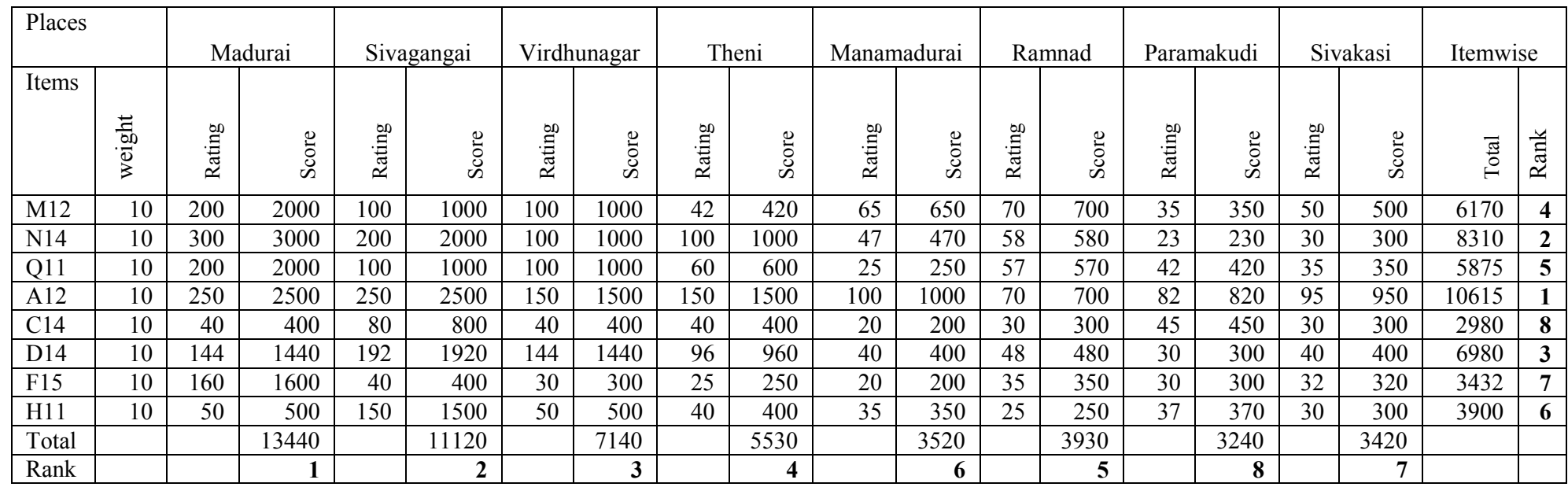

Apply these best two items and places in the fuzzy decision making Algorithms for optimization. The steps for FDM algorithms is given.

STEP 1: Choose the Items to solve the criteria that will be used in the decision making process

STEP 2: Create Places with fuzzy weight for each criterion and give the conjunctions to compose them

STEP 3: Create a matrix by the combination between Places and Items for each decision criterion. These matrices must contain the information about the relation between each Place and each item.

STEP 4: Create the fuzzy conditional statements to represent possible database knowledge

STEP 5: Obtain, for each matrix of STEP 3, the fuzzy set $Z_{i}$ that is formed by the input weight, according to $Z_{i}=\mu_{i}$ $\left(\mathrm{X}_{\mathrm{jk}}\right) / \mathrm{P}_{\mathrm{jk}}$ where $\mathrm{i}, \mathrm{j}$ and $\mathrm{k}$ represent Criterion, Items, and Places respectively. And $\mathrm{P}_{\mathrm{jk}}$ is the weight assigned to the items $\mathrm{j}$ for a place $\mathrm{k}$ in a given criterion $\mathrm{i}$.

STEP 6: Obtain the fuzzy set $\mathrm{L}_{\mathrm{i}}$ formed by the weight $\mathrm{P}_{\mathrm{jk}}$ that are assigned to the pertinence of matrix which, in turn, is given by the ratio between each weight and the largest value among all the weight of the same matrix. The following equations express these value, where $\Lambda$ repersents the largest weight of the matrix,

$$
\mathrm{L}_{\mathrm{i}}=\mu^{\Lambda}\left(\mathrm{X}_{\mathrm{jik}}\right) / \mathrm{P}_{\mathrm{jk}}, \mu^{\Lambda}\left(\mathrm{X}_{\mathrm{jk}}\right)=\left(\mathrm{X}_{\mathrm{jk}}\right) / \Lambda
$$

STEP 7: Obtain from $\mathrm{Zi}$, Li a matrix $\mathrm{Ci}$ that is expressed by the equations,

$$
\mu C^{\Lambda}\left(X_{j k}\right)=\operatorname{Max}\left(\mu_{i}\left(X_{j k}\right), \mu^{\Lambda}\left(X_{j k}\right)\right), C_{i}=\mu C^{\Lambda}\left(X_{j k}\right) / P_{j k}
$$

STEP 8: Use Max to compose the fuzzy decision set, according to STEP 2

STEP 9: Present Final Decision set for each criteria.

Apply the Result of Decision Matrix in FDM Algorithm, it will produce the following result.

\begin{tabular}{|c|c|c|c|c|c|c|c|}
\hline Items (4) - A12 & $\begin{array}{l}\text { Quantity of } \\
\text { sales }\end{array}$ & $\begin{array}{l}\text { Weight } \\
\left(\mu_{\mathrm{i}}\right)\end{array}$ & $\mathbf{Z}_{\mathrm{i}}=\mu_{\mathrm{i}}\left(\mathbf{X}_{\mathrm{jk}}\right) / \mathbf{P}_{\mathrm{jk}}$ & $\begin{array}{l}\mu^{\Lambda} \quad\left(\mathbf{X}_{\mathrm{jk}}\right)= \\
\left(\mathbf{X}_{\mathrm{jk}}\right) / \Lambda\end{array}$ & $\begin{array}{l}\mathbf{L}_{\mathbf{i}}=\mu_{\Lambda} \\
\left(\mathbf{X}_{\mathbf{j k}}\right) / \mathbf{P}_{\mathbf{j k}}\end{array}$ & $\begin{array}{l}\mu C_{\Lambda}\left(X_{j k}\right) \\
=M a x \\
\left(\mu_{i}\left(X_{j k}\right)\right. \\
\left.\mu_{\Lambda}\left(X_{i k}\right)\right)\end{array}$ & $\begin{array}{l}\mathbf{C}_{\mathbf{i}}=\mu \mathbf{C}^{\Lambda}\left(\mathbf{X}_{\mathrm{jk}}\right) \\
/ \mathbf{P}_{\mathbf{j k}}\end{array}$ \\
\hline Madurai & 250 & 1 & 1 & 250 & 1 & 250 & 1 \\
\hline Sivagangai & 250 & 1 & 1 & 250 & 1 & 250 & 1 \\
\hline Virdhunagar & 150 & 1 & 0.6 & 150 & 0.6 & 150 & 0.6 \\
\hline Theni & 150 & 1 & 0.6 & 150 & 0.6 & 150 & 0.6 \\
\hline Mamamadurai & 100 & 1 & 0.4 & 100 & 0.4 & 100 & 0.4 \\
\hline Ramnad & 70 & 0.5 & 0.14 & 70 & 0.28 & 70 & 0.28 \\
\hline Paramakudi & 82 & 0.5 & 0.164 & 82 & 0.328 & 82 & 0.328 \\
\hline
\end{tabular}

Table 3: FDM algorithm for Item 4(A12)

$\mu_{\mathrm{i}}--->=100$ (Sales Quantity) - 1 (Rating)

$\mu_{\mathrm{i}}---<100 \quad$ (Sales Quantity) - 0 (Rating)

$\mathrm{Pjk}=250$ (Maximum in I4)

$\mu \mathrm{Ci}$--- >= 100 (Sales Quantity) - 1 (Rating)

$\mu \mathrm{Ci}---<100 \quad$ (Sales Quantity)- 0 (Rating) 
Table 4: FDM algorithm for Item 2(N14)

\begin{tabular}{|l|c|l|c|c|c|c|c|}
\hline Items(4)-N14 & $\begin{array}{l}\text { Quantity of } \\
\text { sales }\end{array}$ & $\begin{array}{l}\text { Weight } \\
\left(\mu_{\mathbf{i}}\right)\end{array}$ & $\begin{array}{l}\mathbf{Z}_{\mathbf{i}}=\boldsymbol{\mu}_{\mathbf{i}} \\
\left(\mathbf{X}_{\mathbf{j k}}\right) / \mathbf{P}_{\mathbf{j k}}\end{array}$ & $\begin{array}{l}\boldsymbol{\mu}^{\Lambda}\left(\mathbf{X}_{\mathbf{j k}}\right)= \\
\left(\mathbf{X}_{\mathbf{j k}}\right) / \mathbf{\Lambda}\end{array}$ & $\begin{array}{l}\mathbf{L}_{\mathbf{i}}=\mu_{\boldsymbol{\Lambda}}\left(\mathbf{X}_{\mathbf{j k}}\right) / \\
\mathbf{P}_{\mathbf{j k}}\end{array}$ & $\begin{array}{l}\mu \mathbf{C}_{\boldsymbol{\Lambda}}\left(\mathbf{X}_{\mathbf{j k}}\right)=\mathbf{M a x} \\
\left(\boldsymbol{\mu}_{\mathbf{i}}\left(\mathbf{X}_{\mathbf{j k}}\right), \mu_{\boldsymbol{\Lambda}}\left(\mathbf{X}_{\mathbf{j k}}\right)\right.\end{array}$ & $\begin{array}{l}\mathbf{C}_{\mathbf{i}}=\mu \mathbf{C}^{\Lambda}\left(\mathbf{X}_{\mathbf{j k}}\right) / \\
\mathbf{P}_{\mathbf{j k}}\end{array}$ \\
\hline Madurai & 300 & 1 & 1 & 300 & 1 & 300 & 1 \\
\hline Sivagangai & 200 & 1 & 0.6667 & 200 & 0.6667 & 200 & 0.6667 \\
\hline Virdhunagar & 100 & 1 & 0.3333 & 100 & 0.3333 & 100 & 0.3333 \\
\hline Theni & 100 & 1 & 0.3333 & 100 & 0.3333 & 100 & 0.3333 \\
\hline Mamamadurai & 47 & 0.5 & 0.0783 & 47 & 0.1567 & 47 & 0.1567 \\
\hline Ramnad & 58 & 0.5 & 0.0967 & 58 & 0.1933 & 58 & 0.1933 \\
\hline Paramakudi & 23 & 0.5 & 0.0383 & 23 & 0.0767 & 23 & 0.0767 \\
\hline Sivakasi & 30 & 0.5 & 0.05 & 30 & 0.1 & 30 & 0.1 \\
\hline
\end{tabular}

$\mu_{\mathrm{i}}$--- > $=100$ (Sales Quantity) - 1 (Rating)

$\mu_{\mathrm{i}}---<100 \quad$ (Sales Quantity) - 0 (Rating)

$\mathrm{Pjk}=250($ Maximum in I2)

$\mu \mathrm{Ci}$--- > = 100 (Sales Quantity) - 1 (Rating)

$\mu \mathrm{Ci}---<100 \quad$ (Sales Quantity)- 0 (Rating)

\section{Experimental Analysis and Result}

Fuzzy union and fuzzy intersection techniques are applied to find out an efficient decision model which is utilized to acquire successful sales promotion. The calculations are tabulated as follows.

Table54: Fuzzy values for best items A12 and N14

\begin{tabular}{|l|c|c|}
\hline & Item 4- A12 & Item 2-N14 \\
\cline { 2 - 3 } & $\mathbf{C}_{\mathbf{i}}=\boldsymbol{\mu \mathbf { C } ^ { \Lambda } ( \mathbf { X } _ { \mathbf { j k } } ) / \mathbf { P } _ { \mathbf { j k } }}$ & $\mathbf{C}_{\mathbf{i}}=\boldsymbol{\mu \mathbf { C } ^ { \Lambda } ( \mathbf { X } _ { \mathbf { j k } } ) / \mathbf { P } _ { \mathbf { j k } }}$ \\
\hline Madurai & 1 & 1 \\
\hline Sivagangai & 1 & 0.6667 \\
\hline Virdhunagar & 0.6 & 0.3333 \\
\hline Theni & 0.6 & 0.3333 \\
\hline Mamamadurai & 0.4 & 0.1567 \\
\hline Ramnad & 0.28 & 0.1933 \\
\hline Paramakudi & 0.328 & 0.0767 \\
\hline Sivakasi & 0.38 & 0.1 \\
\hline
\end{tabular}

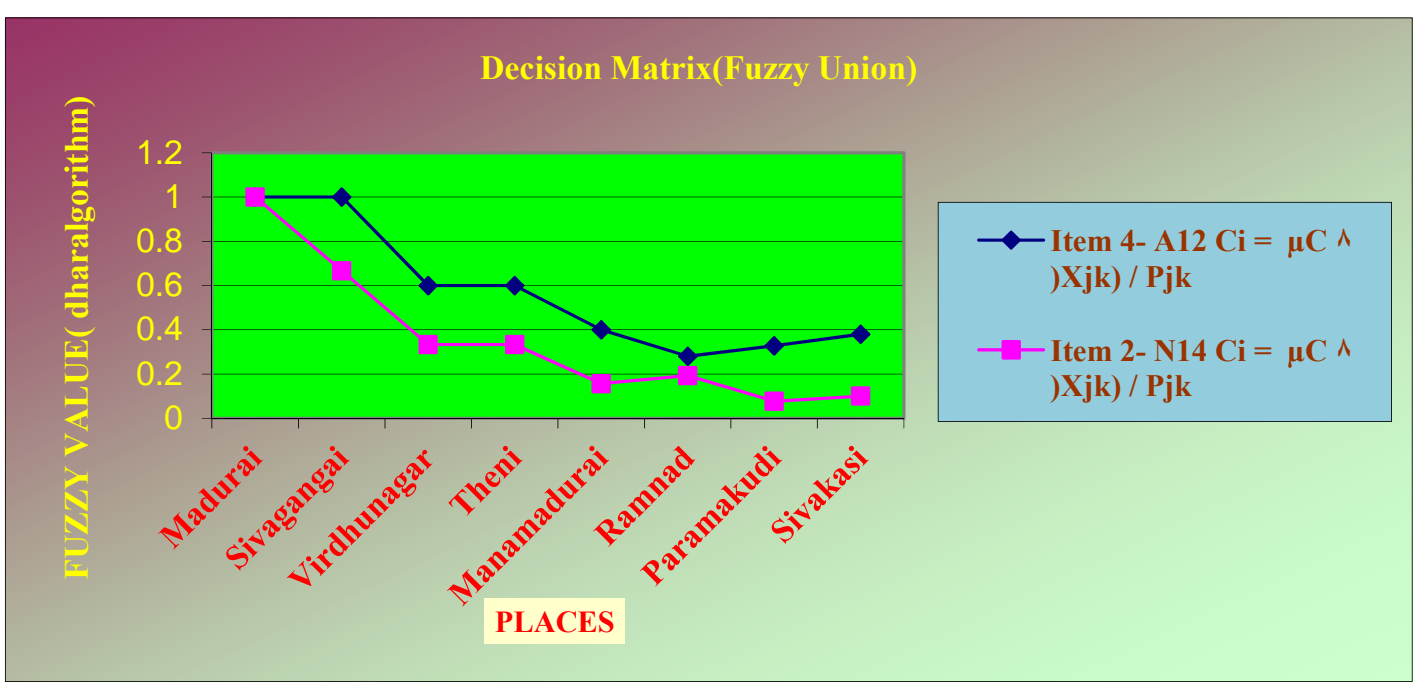

Figure 2:Fuzzy Union for Item I4 and I2 


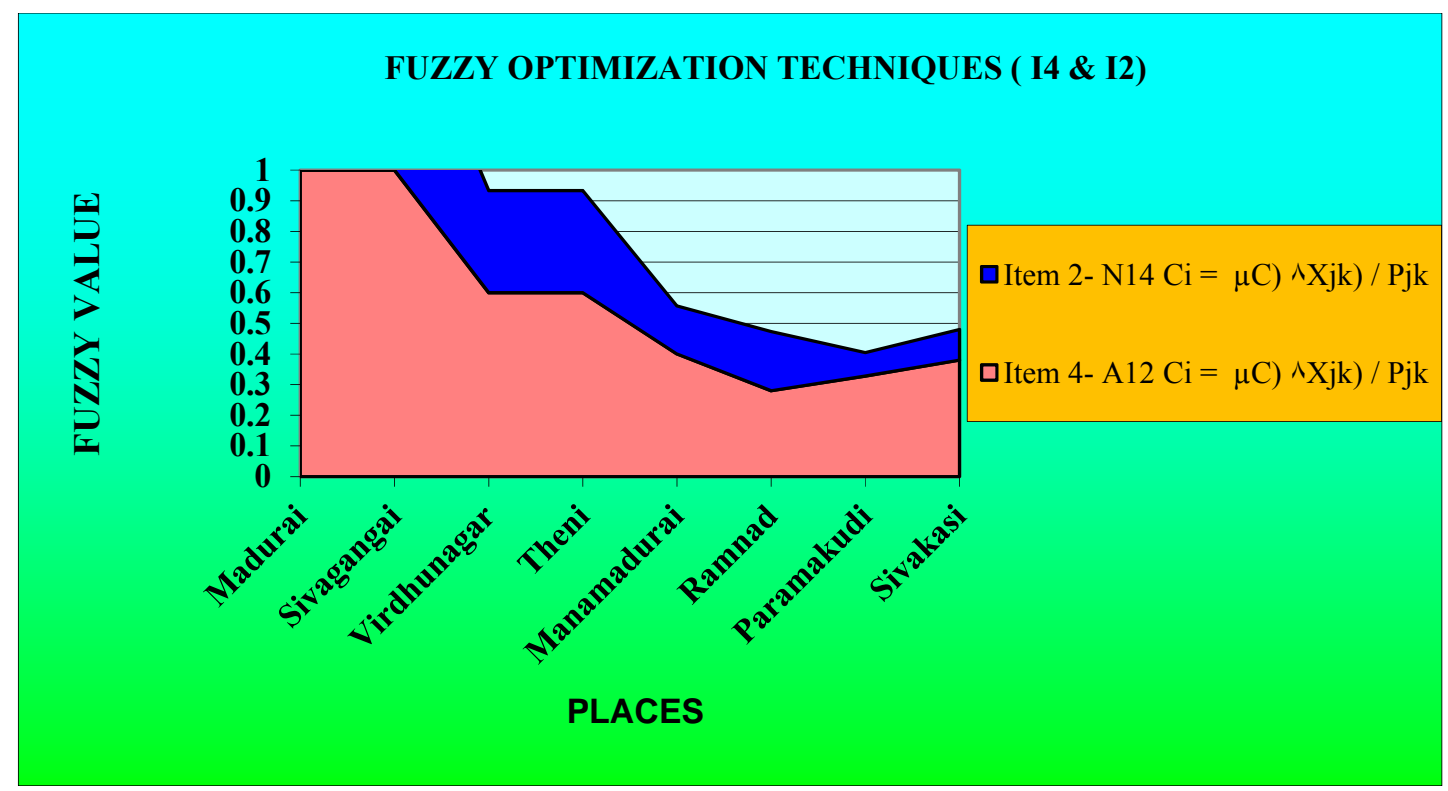

Figure3:Fuzzy Optimization Technique for Item I4 and I

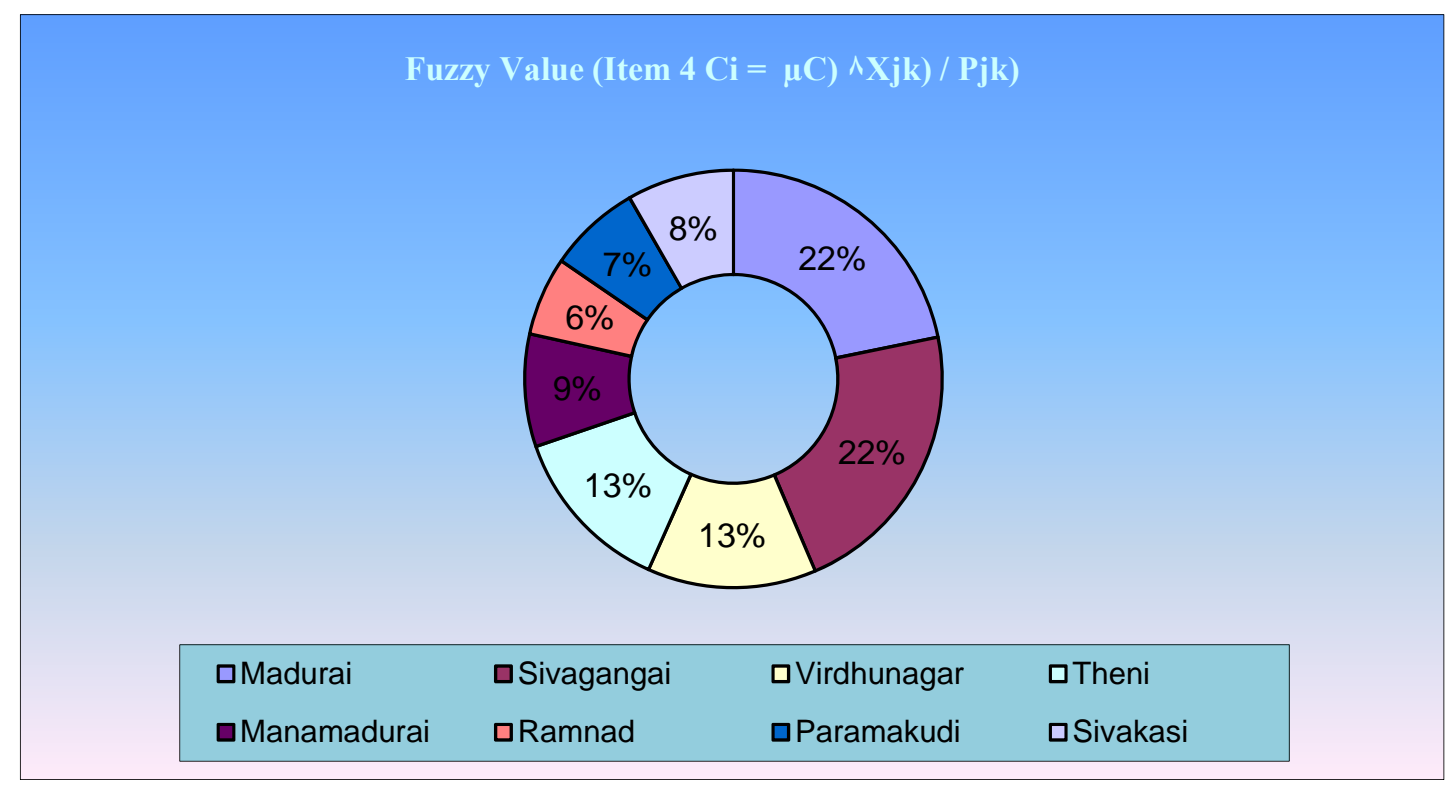

Figure 4:Fuzzy value for Item4

Item 4 (A12) from decision matrix shows maximum sales in all places comparing to Item 2 (N 14) by using Area Chart.

Item 4(A12) Doughnut chart shows that Madurai and Sivagangai city hold $22 \%$ of sales comparing other cities which carry more. 


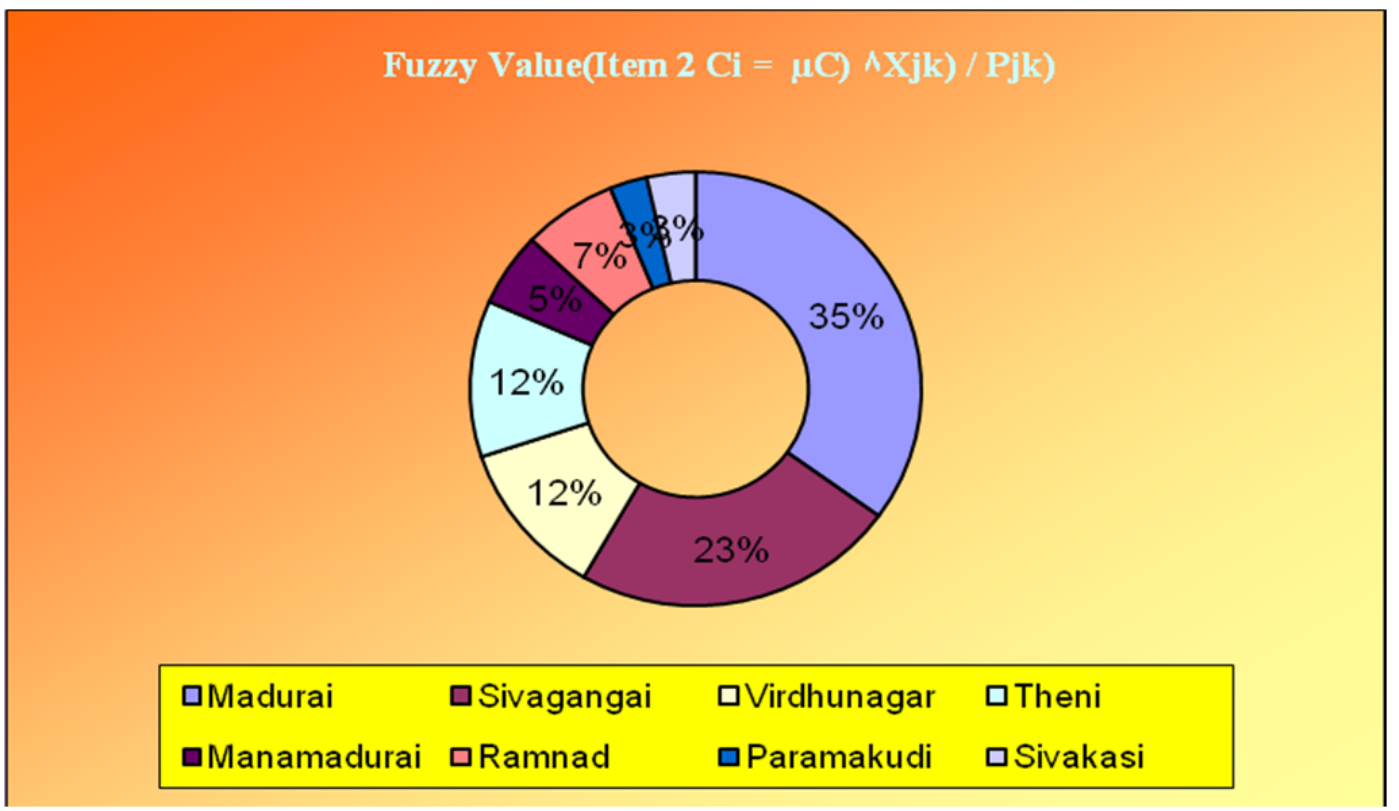

Figure 5: Fuzzy value for Item2

Item 2(N14) Doughnut Chart shows that Madurai holds 35\% of sales comparing to other cities which carry more.

\section{CONCLUSION}

This paper proposes the Fuzzy optimization techniques with FDM algorithm, helps to support and direct strategic on decision making and the setting of business strategies. This algorithm based on decision making which were successfully applied to sale promotion and it was implemented by using FDM algorithm. The decision-making techniques presented here that can help you assist in identifying and finding maximum sales in various places. Finally we present decision matrix and a new FDM algorithm to assess the performance of sales promotion. FDM algorithm which optimizes the maximum sales quantity in various places using decision matrix to show the quantity of sales on different places.

\section{REFERENCES}

[1] D. Fasel and D. Zumstein., A fuzzy data warehouse approach for web analytics. "A Web Science Perspective, volume 5736 of Lecture Notes in Computer Science, page 276-285. Springer, 2009.

[2] Master Data Management An Oracle White Paper September 2011.

[3] Munoz L., Mazon, J., Trujillo, J-, "Systematic review and comparison of modelig ETL processes in data warehouse", Iberian Conference on information Systems and Technologies, June 2010.

[4] Sabir Asadullaev, Data Warehouse Architectures - III SWG IBM EE/A 03.11.2009.

[5] Shaker H. Ali EL - Sappagh a, Abdeltawab M, Ahmed Hendawi b, Ali Hamed El Bastawissy b" A proposed model for data warehouse ETL processes"

[6] Simitsis, A Vassiliadis, P. Sellis, T. -, "State-space optimization of ETL workflows", IEEE Transactions on Knowledge and Data Engineering, Vol 17, Issue 10, Oct 2005.

[7] Inmon, William (2000-07-18). "Data Mart Does Not Equal Data Warehouse". DMReview. com.

[8] Hariprasad T, "ETL testing Fundamentals" on March 29, 2012

[9] Huamin Wang, "An ETL Service Framework Based on Metadata", 2nd International Workshop on Intelligent Systems and Applications, May 2010.

[10] W. H. Inmon. "Building the Data Warehouse". Wiley Publishing, Inc., 4 edition, 2005.

[11] Inmon, William (2000-07-18). "Data Mark Dose Not Equal Data Warehouse". DMReview.com.

[12] Jeffrey R. Bocarsly, Complex ETL Testing-A Strategic Approach

[13] R. Kimball and M. Ross. "The Data Warehouse Toolkit.” Wiley publishing, Inc., 2002.

[14] Li Jian, Xu Bihua, "ETL tool research and implementation based on drilling data warehouse", Seventh International Conference on Fuzzy Systems and Knowledge Discovery, April 2010

[15] Lunan $\mathrm{Li}$, "A framework study of ETL processes optimization based on metadata repository" International Conference on Computer Engineering and Technology, April 2010.

[16] D. Ashok Kumar and M.C. Loraine Charlet Annie," Decision Making on Dichotomous Medical Data using Novel Clustering approach", National conference on Signal and Image processing (NCSIP) 2012.

[17] Daniel Fasel, "A fuzzy data warehouse approach for the customer performance measurement for a hearing instrument manufacturing company", Sixth International conference on fuzzy systems and Knowledge discovery 2009. 
[18] J.F.Baldwin, "Fuzzy systems - logic and reasoning in fuzzy applications".

[19] Jeremy, Jean King and Andreas schindler, "Understanding of Business Intelligence: ETL and Data mark Practises".

[20] Lior Sapir and Armin Shmilovice., "A methodology for the design of a fuzzy data warehouse" 2008.

[21] Owen kaser, Steven Keith and Daniel Lomire, "Dataware housing with literature". September 11, 2006.

[22] Radha Krishna and Sree Kanth, "An Object Oriented modeling and Implementation of Web based ETL process" in IJCSNS, International Journal of Computer Science and Network Security, vol 10 no.2., February 2010.

[23] A. Prema and A. Petha Lakshmi "An Improved Enhancement of Decision - making Analysis to increase the sales promotion using Hyper ETL in Data Mare" in IJCJJ 2012 dec.

[24] A. Prema and A. Petha lakshmi, "An enhanced approach of Hyper ETL to make Efficient Decision Making in Data Mart using Decision Analysis Criteria, IJAIEM July 2013.

[25] Kankana Chakrabarthy "Fuzzy union and intersection of fuzzy set".

[26] A. Prema and A. Petha lakshmi” An approach to Formulate Intelligent Data Repository" in International Journal of Computer Science Trends and Technology (IJCST) - Volume 2 Issue 3, May-Jun 2014.

[27] A.Prema and A.Pethalakshmi,"Novel approach in ETL” in IEEE Xplore” Mar 2013 